\title{
Comparaison de l'efficacité de deux méthodes de multiplication rapide de plants de bananier à partir de l'étude des caractéristiques agronomiques d'un hybride de bananier plantain (Musa spp.)
}

\author{
Léopold SADOM $^{1 *}$, Kodjo TOMEKPÉ ${ }^{1}$, Michel FoLLIOT ${ }^{2}$, François-Xavier CôTE ${ }^{2}$
}

\author{
${ }^{1}$ Programme Amélior. Génét., \\ Cent. Afr. Rech. Banan. \\ Plantains (CARBAP), Njombé, \\ Cameroun, BP 832, Douala, \\ Cameroun, CARBAP, BP 832, \\ Douala, Cameroun \\ mboulaleo@yahoo.fr, \\ sadomleopold@live.fr
}

2 CIRAD-Persyst, UPR Syst. Banan. Ananas, TA B-26 / PS4, Blvd. la Lironde,

F-34398 Montpellier Cedex 5, France

* Correspondance et tirés à part

Fruits, 2010, vol. 65, p. 3-9 (C) 2010 Cirad/EDP Sciences All rights reserved DOI: 10.1051/fruits/2009036 www.fruits-journal.org

RESUMEN ESPAÑoL, p. 9
Comparison of the effectiveness of two vegetative propagation methods for banana trees from the study of the agronomic characteristics of a hybrid of plantain (Musa spp.).

Abstract - Introduction. The objective of our study was to evaluate the agronomic performance of plantain hybrid plants (CRBP-39, Musa cv. AAAB) obtained through two vegetative propagation methods: in vitro culture, called the "CIV technique", and a newly developed technique of plants derived from stem bits, called the "PIF technique". The study, carried out in Cameroon, took place at a high altitude $(1250 \mathrm{~m})$ and covered one cropping cycle. The CRBP-39 hybrid was chosen because of its resistance to Black Leaf Streak Disease and its agronomic performance. Materials and methods. Vitroplants and plants obtained through the PIF technique developed at CARBAP (Cameroon) were planted separately in elementary plots of 16 plants. No chemical control was administered against Black Leaf Streak Disease. Observations were essentially made on agronomic characteristics. Results and discussion. For the mother crop, there was no significant difference between the plants obtained from one or the other vegetative propagation method concerning their pseudo-stem height and girth; the number of functional leaves at shooting; the duration of the production cycle; the bunch weight; the number of hands and fruits of the bunch; and the length and circumference of the fruit. For the ratoon crop, significant differences were observed concerning the pseudo-stem height and girth, and the number of emitted and functional leaves at harvest. These results globally indicate a strong similarity in vegetative and bunch characteristics of plants propagated with one or the other method. The PIF technique, which is used more and more by small-scale farmers because of its simplicity, rapidness and low cost of production, is a method of vegetative propagation that helps to produce plants similar to tissue cultured plants in terms of agronomic qualities.

Cameroon / Musa (plantains) / hybrids / plant propagation / vitroplants / propagation materials

Comparaison de l'efficacité de deux méthodes de multiplication rapide de plants de bananier à partir de l'étude des caractéristiques agronomiques d'un hybride de bananier plantain (Musa spp.).

Résumé - Introduction. Nos travaux ont eu pour objectif de comparer les performances agronomiques des plants d'un hybride de bananier plantain (CRBP-39, Musa cv. AAAB) obtenus par utilisation de deux méthodes de multiplication végétative: la culture de tissus in vitro (CIV) et une technique de propagation par multiplication de plants à partir de fragments de tiges (dite technique des PIF) développée récemment. L'étude, effectuée au Cameroun, a été menée en altitude $(1250 \mathrm{~m})$; elle a porté sur un cycle de culture. L'hybride CRBP-39 a été choisi en raison de sa résistance à la maladie des raies noires et de ses performances agronomiques. Matériel et méthodes. Des vitroplants et des plants obtenus par la technique des PIF, technique de propagation horticole développée au CARBAP (Cameroun), ont été plantés séparément en blocs, dans des parcelles de terrain élémentaires de 16 plants. Aucun traitement chimique n'a été appliqué contre la maladie des raies noires. Les observations ont été faites essentiellement sur des caractéristiques agronomiques. Résultats et discussion. Pour le pied-mère, il n'y a eu aucune différence significative entre les plantes obtenues à partir de l'une ou de l'autre méthode de multiplication végétative quant à la taille et au périmètre de la pseudo-tige ; le nombre de feuilles fonctionnelles à la floraison ; la durée du cycle de production; le poids de régime ; le nombre de mains et de fruits du régime; la longueur et la circonférence du fruit. Pour le rejet successeur, il y a eu des différences significatives de taille et de périmètre de la pseudo-tige, ainsi que du nombre de feuilles émises et fonctionnelles à la récolte. Ces résultats montrent globalement une grande similitude des caractéristiques agronomiques des plants issus des deux méthodes de multiplication végétative. La technique des PIF, de plus en plus utilisée compte tenu de sa simplicité, son faible coût de production et sa rapidité de mise en ouvre, est donc une technique horticole qui permet de produire des plants de qualité agronomique comparable aux vitroplants.

Cameroun / Musa (banane plantain) / hybride / multiplication des plantes / vitroplant / matériau de multiplication 


\section{Introduction}

Les plantains (Musa spp., sous-groupe AAB) constituent une denrée de base dans les zones forestières humides de l'Afrique centrale et occidentale où ils représentent $60 \%$ à $70 \%$ de la production mondiale [1].

Cependant, cette culture est soumise à des contraintes parasitaires parmi lesquelles la maladie des raies noires (MRN), provoquée par le champignon pathogène Mycosphaerella fijiensis, considérée comme la plus grave des maladies fongiques affectant la production bananière [2]. Les pertes dues à cette maladie touchent $20 \%$ à $80 \%$ [3] des récoltes et tous les cultivars de plantain locaux peuvent être touchés. Ainsi, la création de variétés résistantes par hybridation contrôlée est apparue être l'une des meilleures technologies pour contrôler cette maladie.

Depuis plusieurs années, le programme d'amélioration génétique du Centre Africain de Recherches sur Bananiers et Plantains (CARBAP, Cameroun) a concentré ses efforts dans cette voie. L'hybride de type plantain tétraplö̈de 'CRBP-39' ( Musa cv. $\mathrm{AAAB}$ ), obtenu par croisement entre un plantain femelle triploïde (Musa cv. AAB, variété locale 'French clair') et un bananier mâle diploïde M53, hybride synthétique (AA), est l'un des résultats de ce travail. Des études sur les performances agronomiques et la résistance à la maladie des raies noires de cet hybride 'CRBP-39' multiplié par culture in vitro ont eu pour objectifs de confirmer ses performances et de déterminer les pertes de rendement imputables à la maladie des raies noires par comparaison avec celles de son parent plantain femelle 'French clair' traité ou non contre cette maladie dans les conditions pédo-climatiques du centre de recherche du CARBAP [4].

Toutefois, il se pose un problème crucial de disponibilité de matériel végétal sain en quantité suffisante. Les rejets sont souvent peu homogènes du point de vue physiologique et, de plus, ils sont fréquemment contaminés par le charançon noir et les nématodes. Cependant, l'utilisation de la culture in vitro (CIV) et les méthodes horticoles de multiplication rapide permettent de lever, dans une certaine mesure, cette contrainte [5]. Ces techniques de culture de tissus in vitro permettent de produire de grandes quantités de plants. Malheureusement, les coûts élevés de production des plants par CIV ne sont pas toujours accessibles aux petits producteurs qui sont régulièrement confrontés au problème de disponibilité des plants. Dans ce contexte, le CARBAP a mis au point une nouvelle technique de multiplication de masse in vivo, la technique des " Plants Issus de Fragments de tige ", qui permet d'activer des bourgeons latents et de produire rapidement, hors du sol, d'importantes quantités de matériel de plantation sain [6]. Notre travail a comparé les performances agronomiques des plants de cet hybride CRBP-39 obtenus à partir de ces deux techniques de multiplication : technique de culture in vitro (CIV) et technique des "Plants Issus des Fragments de tige ".

\section{Matériel et méthodes}

Le matériel végétal testé a été constitué de rejets de l'hybride CRBP-39 (génome AAAB), résistant à la maladie des raies noires et doté de bonnes caractéristiques agronomiques.

Deux lots de plants obtenus par multiplication végétative intensive ont été utilisés :

- Un premier lot de bananiers a été constitué de 180 plants issus de culture in vitro (CIV) à partir de bourgeonnements axillaires. Le démarrage de la culture s'est fait à partir de trois rejets de l'hybride CRBP-39 multipliés par la société Vitropic S.A. (SaintMatthieu-de-Tréviers, France).

- Un second lot de bananiers a été obtenu, au Cameroun, par la technique des "Plants Issus de Fragments de tige" (PIF) qui permet d'activer des bourgeons latents sur les tiges et de produire rapidement, hors du sol, des quantités très importantes de plants sains [6]. Après arrachage des rejets de CRBP-39 au champ, les tiges ont été lavées et débarrassées de leurs racines et de toute partie nécrosée. Les bourgeons ont été rendus accessibles par "déshabillage " ou 
" décorticage " de la tige, c'est-à-dire par enlèvement au couteau de toutes les gaines foliaires présentes. Les fragments de tiges obtenus ont ensuite été mis à sécher à l'ombre et dans un endroit sec pendant (24 à 48) h avant d'être placés dans un germoir spécialement aménagé à cet effet [6]. Le sevrage des premiers plants, munis d'au moins six feuilles vivantes, a eu lieu trois à cinq semaines plus tard. Les jeunes bananiers ont alors été sortis du germoir et repiqués sous ombrière dans des sacs de polyéthylène noirs et opaques, d'une contenance de $0,5 \mathrm{~L}$ et remplis d'un mélange [1 volume de terre +1 volume de parche de café] stérilisé au feu de bois pendant au moins $12 \mathrm{~h}$. Les plants sevrés ont été mis en terre dans une parcelle expérimentale.

Notre étude a porté sur le premier cycle de culture. La parcelle expérimentale a été mise en place au Cameroun en mai 2004 dans la station de Mbouroukou (alt. $1250 \mathrm{~m}$, lat. $5^{\circ} 01^{\prime} \mathrm{N}$, long. 9० $17^{\prime} \mathrm{E}$, située au versant du Mont Manengouba). Les sols de cette parcelle sont des andosols de type volcanique. Le climat est de type tropical humide à deux saisons : une saison humide d'avril à octobre et une saison sèche de novembre à mars.

La plantation a été réalisée avec des écartements de $3 \mathrm{~m}$ entre les lignes $\times 2 \mathrm{~m}$ sur la ligne. Le dispositif expérimental a été un modèle complètement randomisé avec des parcelles élémentaires de 16 plants, chacune d'elles séparée par deux lignes du cultivar Grande Naine afin d'éviter des effets de bordures. Onze parcelles de plants issus de CIV, soit 176 plants, et cinq parcelles de plants issus de PIF, soit 80 plants, ont été suivies individuellement. La parcelle expérimentale n'a pas été traitée contre la maladie des raies noires et les deux types de plants (issus de CIV ou issus de PIF) ont été menés avec des techniques culturales classiques; les traitements insecticide et nématicide ont été appliqués de façon uniforme [7].

Un certain nombre de caractéristiques agronomiques a été mesuré tout au long du cycle de culture :

- La hauteur et la circonférence du piedmère et celle du plus grand rejet successeur ont été évaluées à $1 \mathrm{~m}$ du sol ; l'intervalle en jours, entre la plantation et la floraison (IPF) a été calculé, et le nombre de feuilles vivantes a été comptabilisé à la floraison.

- L'intervalle en jours, entre la plantation et la coupe (IPC), et entre la floraison et la coupe (IFC), le poids du régime, le nombre de mains par régime, le nombre de doigts par régime, la longueur et la circonférence du doigt médian situé sur la deuxième main du régime ont été évalués à la récolte.

Les données ont été traitées par analyse de variance à une seule entrée sans bloc à $5 \%$ de signification à l'aide du logiciel Genstat. Les moyennes ont été séparées par le test de LSD (Least Significant Difference).

\section{Résultats et discussion}

L'analyse des caractéristiques végétatives et de certaines caractéristiques des régimes en fonction de la technique de multiplication utilisée montre que, quelle que soit cette technique, la hauteur du pseudo-tronc du pied mère à la floraison n'est pas significativement différente d'un lot de plants à l'autre (tableau I).

Par ailleurs, les plants obtenus par CIV et PIF ont bien exprimé les caractéristiques variétales de l'hybride CRBP-39 en ce qui concerne la hauteur qui, elle-même, n'a pas présenté de différences significatives d'un type de plant à l'autre. La taille du pseudotronc du bananier plantain est un caractère important. La taille idéale devrait être inférieure à $3 \mathrm{~m}$ [8] afin de réduire les risques de chute dus au vent et de faciliter la gestion de la plantation ; en particulier, un tuteurage facile et une récolte graduelle des mains et fruits sur pied-mère sont autant d'avantages liés à la stature de la plante [8-11].

La hauteur et la circonférence du plus grand rejet successeur (rejet le plus développé à la floraison) ont présenté des différences significatives entre les plants issus de CIV et ceux issus de PIF (tableau I, figure 1). Les valeurs moyennes obtenues témoignent de la robustesse des plants, caractère intéressant qui leur permet de 


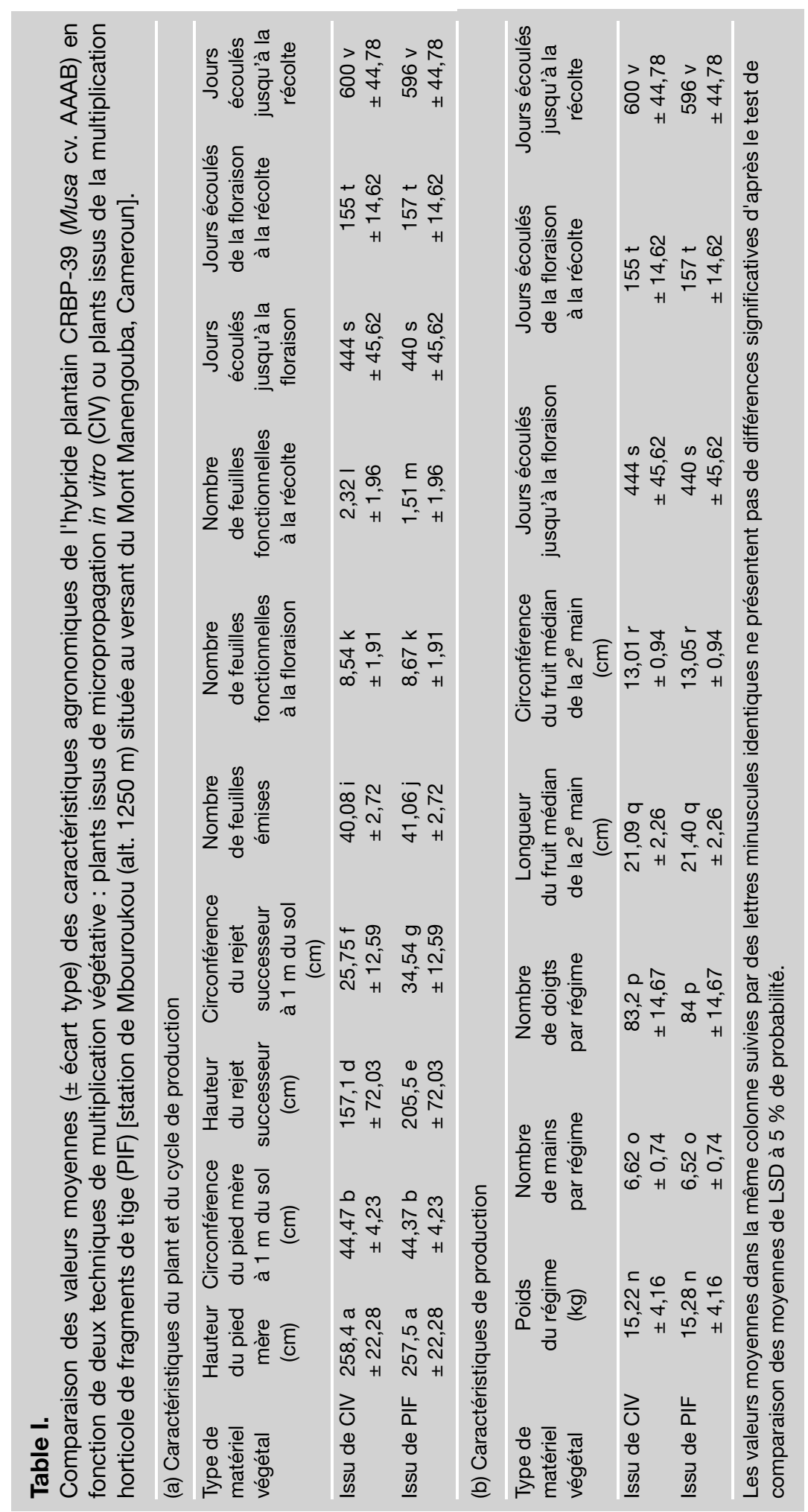


soutenir des grands régimes et de résister aux cassures provoquées par des vents violents.

Le nombre de feuilles émises a été significativement différent entre les plants issus de CIV et de PIF. Les plants issus de PIF produiraient en moyenne davantage de feuilles que ceux issus de CIV (tableau I).

Le nombre moyen de feuilles fonctionnelles à la floraison a été de 8 feuilles pour l'une et l'autre des techniques de multiplication utilisées, ce qui a permis d'une manière générale un bon remplissage des fruits. Un nombre élevé de feuilles fonctionnelles à la floraison est une caractéristique essentielle pour assurer un bon développement du régime et des fruits de qualité [4]. Certains plants issus de CIV et de PIF ont présenté jusqu'à 13 et 14 feuilles fonctionnelles à la floraison.

La récolte du régime a été faite dès le changement de la couleur verte à la couleur jaune de la pelure d'un doigt. Un grand nombre de feuilles vivantes présentes sur le plant entre la floraison et la récolte permet un meilleur remplissage des fruits, des régimes plus lourds et un rendement accru. À la récolte, le nombre de feuilles fonctionnelles a été significativement différent selon les deux types de plants. Les plants issus de CIV ont présenté en moyenne davantage de feuilles vivantes à la récolte que les plants issus de PIF (tableau I). Cependant, ce nombre a été faible, ce qui pourrait être dû à la durée plus importante du cycle de culture des zones d'altitude par rapport aux zones de plaine. En effet, des travaux conduits à Njombé (altitude de $80 \mathrm{~m}$ au dessus du niveau de la mer) portant sur l'évaluation d'hybrides tétraploïdes de bananiers plantains (Musa spp.) résistants à la maladie des raies noires et créés au Cameroun ont montré un nombre de feuilles vivantes à la récolte supérieur (3,8 feuilles en moyenne au second cycle) à celui observé dans notre étude menée en altitude [12]. Aucun traitement fongicide n'ayant été effectué dans notre parcelle d'expérimentation, la résistance des hybrides de plantains vis-à-vis de la maladie des raies noires a été confirmée par l'observation d'un nombre de feuilles à la récolte plus élevé que celui des variétés de plantains traditionnelles [12].

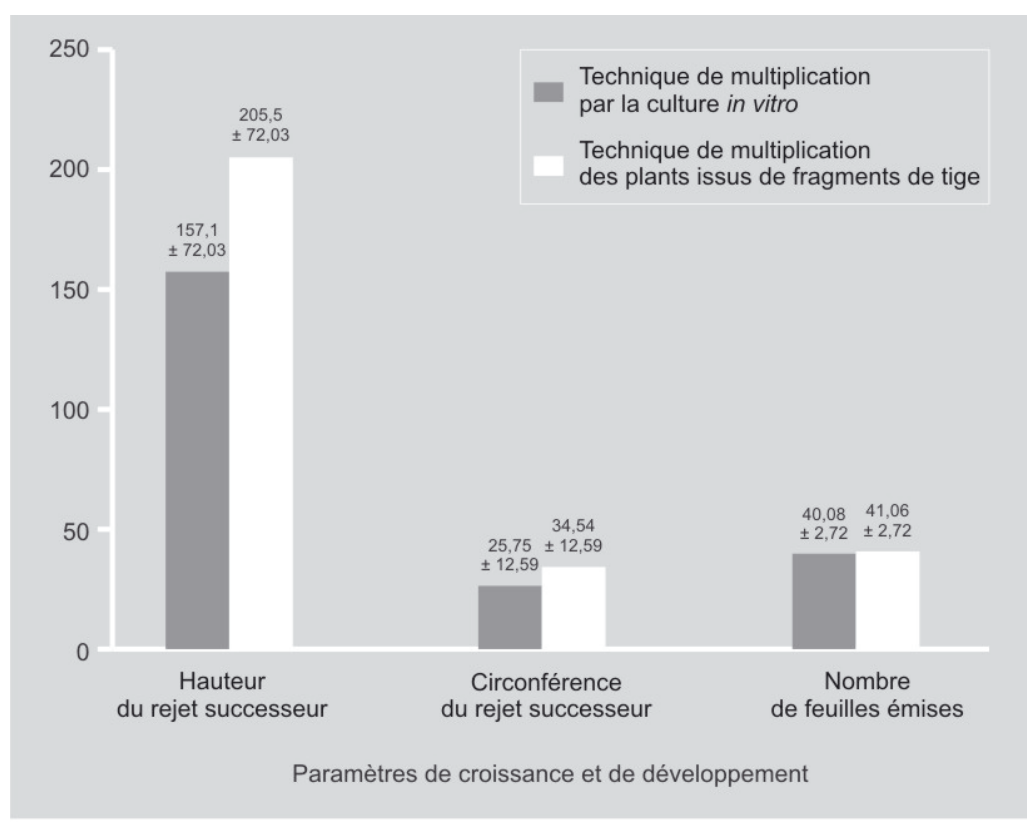

La longueur du cycle de production n'a présenté aucune différence significative entre les plants issus de CIV et ceux issus de PIF. Les plants les plus précoces ont fleuri entre $347 \mathrm{j}$ après la plantation (soit 11,56 mois) pour les plants issus de CIV et $353 \mathrm{j}$ (soit 11,76 mois) pour les plants issus de PIF. En revanche, pour les plants qui ont fleuri tardivement, la durée moyenne entre la plantation et la floraison a été de $440 \mathrm{j}$. Ces résultats témoignent d'une influence de l'altitude sur la date de floraison mise en évidence lors de notre étude, par rapport à d'autres observations effectuées sur des plants de CRBP-39 issus de CIV et conduits en plaine [4, 12]. Une floraison précoce couplée à une taille réduite des plants sont des caractéristiques susceptibles de diminuer les dégâts dus aux vents [13].

Il a fallu en moyenne $155 \mathrm{j}$ (soit 5,16 mois) pour que les premiers fruits des deux types de plants virent à la couleur jaune. Aucune différence n'a été mise en évidence dans le poids du fruit, le nombre de mains, le nombre de fruits ou doigts, la longueur du fruit ou la grosseur du fruit médian chez les deux catégories de plants. Le poids moyen des
Figure 1.

Comparaison des paramètres agronomiques de plants issus de deux types de technique de multiplication (CIV et PIF) de I'hybride de plantain 'CRBP39'. Les valeurs moyennes sont présentées avec leur écart-type. Pour ces trois paramètres, les différences entre les plants de l'une ou l'autre origine ont été significatives. 
régimes a été de $15 \mathrm{~kg}$ pour les deux catégories de plants, avec une moyenne de 120 fruits pour les plants issus de CIV et de 97 fruits pour les plants issus de PIF. La longueur maximale des doigts médians a été de $27 \mathrm{~cm}$ pour un diamètre (grade) de $16 \mathrm{~cm}$. Globalement, il y a une grande similitude des caractéristiques végétatives et des caractéristiques de régimes des deux catégories de plants analysés. Il n'y a donc pas eu de différence significative de productivité. La technique des PIF appliquée à un matériel dont on a contrôlé la qualité sanitaire pourrait donc être utilisée à petite échelle par les petits producteurs disposant de faibles moyens de production. En outre, nos résultats ont confirmé les excellentes performances agronomiques de l'hybride CRBP-39. Ils sont en accord avec ceux de Vulysteke et al. selon lesquels les plants issus des bourgeons axillaires présenteraient les mêmes caractéristiques agronomiques que ceux issus de bourgeons caulinaires [14].

\section{Conclusion}

Notre expérimentation a mis en évidence un retard de floraison et de maturation des fruits lors du premier cycle de production des bananiers, qui pourrait être dû à l'altitude de la parcelle de l'étude $(1250 \mathrm{~m})$. Toutefois, dans le contexte agro-pédologique de Mbouroukou, les différences de croissance à la floraison et à la récolte entre les deux types de plants n'ont pas engendré de différences de rendement.

\section{Remerciements}

Ce travail a été co-financé par le CARBAP et le CIRAD. Les auteurs remercient particulièrement B. Kengni, W. Nguefack, M. Nguidjoï et T. Emadion (CARBAP, Cameroun) pour leur appui technique. Ils remercient également la société Vitropic (Saint-Matthieu de Tréviers, France) pour la fourniture du matériel végétal issu de culture in vitro.

\section{Références}

[1] Rowe P., Réponse d'un sélectionneur à l'article sur le "Programme mondial pour l'amélioration des Musa (PROMUSA)," InfoMusa 7 (1998) I-IV.

[2] Stover R.-H., Buddenhagen I.-W., Banana breeding, polyploidy disease resistance and productivity, Fruits 41 (1986) 175-191.

[3] Dzomeku B.M., Armo-Annor, Akyeampong E., Baning I.S., Farmers evaluation of four Musa hybrids in Ghana, MusAfrica 17 (2006) 12-14.

[4] Cohan J.-P., Abadie C., Tomekpé K., Tchango Tchango J., Performances agronomiques et résistances à la maladie des raies noires de l'hybride 'CRBP-39', InfoMusa 12 (2003) 29-32.

[5] Auboiron E., Le sevrage des vitroplants de bananiers, CRBP, Fiche technique, Njombé, Cameroun, 1996, 3 p.

[6] Kwa M., Activation de bourgeons latents et utilisation de fragments de tige du bananier pour la propagation en masse de plants en conditions horticoles in vivo, Fruits 58 (2003) 315-328.

[7] Noupadja P., Tchango Tchango J., Tomekpé K., Abadie C., Évaluation de cultivars exotiques de bananiers au Cameroun, Cah. Agric. 10 (2001) 19-24.

[8] Jones D., Première réunion du réseau des sélectionneurs de Musa, InfoMusa 3 (1) (1994) 5-9.

[9] Simmonds N.W., Classification and breeding of bananas, in: Persley G.J., De Langhe E.A. (Eds.), Banana and plantain breeding strategies, Proc. Workshop held at Cairns, Aust., 13-17 Oct., 1986, ACIAR Proc. 21 (1987) 69-73.

[10] Tomekpé K., Noupadja P., Mbakwa F.-N., Performances agronomiques des premiers hybrides de plantains (Musa spp.) résistants à la maladie des raies noires, Biosci. Proc. 5 (1998) 46-52.

[11] Tomekpé K., Noupadja P., Abadie C., Auboiron E., Tchango Tchango J., Genetic improvement of plantains at CRBP: Performance of 
black Sigatoka resistant plantain hybrids, in: Giraldo Cardona M.J., Belalcazar Carvajal S.L., Cayon Salinas D.G., Botero Isaza R.G. (Eds.), Proc. Int. Semin. Plantain Prod., Armenia, Quindio, Colombia, 4-8 May, 1998, pp. $45-50$.

[12] Noupadja P., Tomekpé K., Youmbi E., Évaluation d'hybrides tétraploïdes de bananiers plantains (Musa spp.) résistants à la maladie des raies noires créés au Cameroun, Fruits 62 (2007) 77-88.
[13] Vulysteke D., Makumbi D., Ortiz R., Performance of IITA plantain and banana hybrids in Uganda, MusAfrica 9 (1996) 21-23.

[14] Vulysteke D., Swennen R., Ortiz R., Genetic improvement of plantains at the International Institute of Tropical Agriculture (IITA), in: Ganry J. (Ed.), Breeding banana and plantain for resistance to diseases an pests, Proc. Int. Symp. held at Montpellier, 7-9 Sept., 1992, CIRAD-INIBAP, Montpellier, France, 1993, pp. 267-268.

\section{Comparación de la eficacia de dos métodos de multiplicación rápida de plantones de banano, a partir del estudio de las características agronómicas de un híbrido de banano plátano (Musa spp.).}

Resumen - Introducción. Nuestros estudios tuvieron como objetivo la comparación de los resultados agronómicos de los plantones de un híbrido de banano plátano (CRBP-39, Musa cv. AAAB), obtenidos mediante empleo de dos métodos de multiplicación vegetativa: el cultivo de tejidos in vitro (CIV) y una técnica de propagación basada en la multiplicación de plantones a partir de fragmentos de ramas (llamada: técnica PIF), desarrollada recientemente. El estudio, llevado a cabo en Camerún, se realizó en altitud $(1250 \mathrm{~m})$; se refirió a un ciclo de cultivo. Se eligió el híbrido CRBP-39 por causa de su resistencia a la enfermedad de las rayas negras y de sus resultados agronómicos. Material y métodos. Tanto vitroplantones como plantones obtenidos mediante la técnica PIF, técnica de propagación hortícola desarrollada en el CARBAP (Camerún), se plantaron separadamente en bloques, en parcelas de terreno elementares de 16 plantas. Ningún tratamiento químico se aplicó contra la enfermedad de las rayas negras. Se realizaron las observaciones esencialmente sobre las características agronómicas. Resultados y discusión. En cuanto a la planta-madre, entre las plantas obtenidas a partir de un método de multiplicación vegetativa u otro, no hubo ninguna diferencia significativa en relación con el tamaño y el perímetro de la seudo-rama; ni con el número de hojas funcionales en la floración; ni con la duración del ciclo de producción; ni con el peso de régimen; ni con el número de manos y de frutos del régimen; ni con la longitud y la circunferencia del fruto. Para el vástago sucesor, existieron diferencias significativas de tamaño y de perímetro de la seudo-rama, así como del número de hojas emitidas y funcionales en la cosecha. Dichos resultados muestran globalmente una gran similitud de las características agronómicas de los plantones pertenecientes a ambos métodos de multiplicación vegetativa. La técnica PIF, cada vez más empleada gracias a su simplicidad, a su bajo coste de producción y a su rapidez de puesta en ejecución, resulta, por lo tanto, una técnica hortícola que permite la producción de plantones de calidad agronómica comparable a la de los vitroplantones.

Camerún / Musa (plátano) / híbridos / propagación de plantas / vitroplantas / materiales de propagación 